TecnoHumanismo. Revista Científica

Junio - Agosto 2021

Volumen 1 / No.2

ISSN: $2710-2394$

pp. $38-53$

https://doi.org/10.53673/th.v1i10.59

https://tecnohumanismo.online

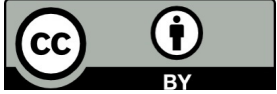

\title{
Correlación entre la resistencia real del concreto y el ensayo no destructivo de esclerometría para muestras de concreto en el departamento de Lambayeque - Perú
}

Correlation between the real strength of concrete and the non-destructive sclerometry test for concrete samples in the department of Lambayeque - Peru

Correlação entre a resistência real do concreto e o ensaio de esclerometria não destrutiva para amostras de concreto no departamento de Lambayeque - Peru

\section{ARTÍCULO ORIGINAL}

Manuel Alejandro Borja Suárez

https://orcid.org/0000-0002-6532-4976

mborja@unprg.edu.pe

Universidad Nacional Pedro Ruiz Gallo, Lambayeque - Perú

Recibido 18 de Enero 2021 | Arbitrado y aceptado 10 de Marzo 2021 | Publicado en 05 Junio 2021

\section{RESUMEN}

La presente investigación busca encontrar el nivel de correlación entre la resistencia real del concreto $(\mathrm{kg} / \mathrm{cm} 2)$ y el ensayo no destructivo de esclerometría, pero sobre todo determinar el nivel de confianza con el que se deberían aceptar estos resultados. Para esto se evaluaron probetas provenientes de diferentes obras de construcción en la región Lambayeque, indistintamente de sus características de diseño, $\mathrm{y}$ antes que sean ensayadas en la prensa de compresión, se les sometió a un examen con esclerómetro digital siguiendo el procedimiento de la NTP 339.181 (2013). Se ensayaron también probetas con diseño controlado para f'c: 175,210 y $280 \mathrm{~kg} / \mathrm{cm} 2$ y así comparar si con estas probetas se obtiene una mejor correlación respecto a las probetas provenientes al azar de diferentes obras. Para las muestra analizadas de todas las obras, se encontró una desviación estándar de $45 \mathrm{~kg} / \mathrm{cm} 2$ (ligeramente alta); y para el caso de concretos de diseño controlado: $13 \mathrm{~kg} / \mathrm{cm} 2$, con un coeficiente de correlación de r2 $=0.9467$ (a los 28 días). Después de procesar todos los datos, se llegó a la conclusión que el ensayo de esclerometría si se puede utilizar para estimar la resistencia a la compresión del concreto pero bajo ciertas condiciones controladas y teniendo en cuenta un margen de error y confiabilidad. Finalmente, se puede concluir que para un nivel de confiabilidad del $95 \%$ la resistencia del concreto obtenida con el esclerómetro podría ser estimada con el siguiente rango de precisión: f'c $\pm 25 \mathrm{~kg} / \mathrm{cm} 2$.

Palabras clave: Concreto, resistencia a la compresión, esclerometría.

\section{ABSTRACT}

The present investigation seeks to find the level of correlation between the actual strength of concrete $(\mathrm{kg} / \mathrm{cm} 2)$ and the non-destructive sclerometric test, but above all to determine the level of confidence with which these results should be accepted. For this, specimens from different construction works in the Lambayeque region were evaluated, regardless of their design characteristics, and before they are tested in the compression press, they were subjected to an examination with a digital sclerometer following the procedure of NTP 339.181 (2013). Specimens with controlled design were also tested for f'c: 175, 210 and $280 \mathrm{~kg} / \mathrm{cm} 2$ and thus compare whether with these specimens a better correlation is obtained with respect to the specimens from random from different works. For the analyzed samples of all works, a standard deviation of $45 \mathrm{~kg} / \mathrm{cm} 2$ was found (slightly high); and for the case of controlled design concretes: $13 \mathrm{~kg} / \mathrm{cm} 2$, with a correlation coefficient of $\mathrm{r} 2=0.9467$ (at 28 days). After processing all the data, it was concluded that the sclerometry test can be used to estimate the compressive strength of concrete but under certain controlled conditions and taking into account a margin of error and reliability. Finally, it can be concluded that for a reliability level of $95 \%$, the strength of the concrete obtained with the sclerometer could be estimated with the following precision range: $\mathrm{f}^{\prime} \mathrm{c} \pm 25 \mathrm{~kg} / \mathrm{cm} 2$.

Keywords: Concrete, compressive strength, sclerometry.

\section{RESUMO}

A presente investigação busca encontrar o nível de correlação entre a resistência real do concreto $(\mathrm{kg} / \mathrm{cm} 2)$ e o ensaio esclerométrico não destrutivo, mas acima de tudo, determinar o nível de confiança com o qual esses resultados devem ser aceitos. Para isso, foram avaliados corpos de prova de diferentes obras da região de Lambayeque, independentemente de suas características de projeto, e antes de serem ensaiados na prensa de compressão, foram submetidos a exame com esclerômetro digital seguindo o procedimento da NTP 339.181 (2013). . Amostras com desenho controlado também foram testadas para f'c: 175,210 e $280 \mathrm{~kg} / \mathrm{cm} 2$ e, portanto, compare se com essas amostras uma melhor correlação é obtida em relação às amostras aleatórias de trabalhos diferentes. Para as amostras analisadas de todas as obras, foi encontrado um desvio padrão de $45 \mathrm{~kg} / \mathrm{cm} 2$ (ligeiramente alto); e para o caso de concretos de delineamento controlado: $13 \mathrm{~kg} / \mathrm{cm} 2$, com coeficiente de correlação de r2 $=0,9467$ (aos 28 dias). Após o processamento de todos os dados, concluiu-se que o teste de esclerometria pode ser utilizado para estimar a resistência à compressão do concreto, mas sob certas condições controladas e levando em consideração uma margem de erro e confiabilidade. Por fim, pode-se concluir que para um nível de confiabilidade de $95 \%$, a resistência do concreto obtida com o esclerômetro pôde ser estimada com a seguinte faixa de precisão: f'c $\pm 25 \mathrm{~kg} / \mathrm{cm} 2$.

Palavras-chave: Concreto, resistência à compressão, esclerometria. 


\section{Introducción}

Actualmente, la forma más frecuente de verificar la calidad de un concreto consiste en obtener muestras de la mezcla fresca en probetas cilíndricas estándar, curadas durante 28 días y someterlas al ensayo de compresión; así obtenemos el f’c que representa la calidad más cercana del concreto puesto en obra.

Para el caso de concreto endurecido en vigas, columnas, zapatas, y cualquier otro elemento estructural, debemos extraer un núcleo mediante discos diamantados, con la desventaja de que afectamos el área efectiva de la sección transversal de concreto y en muchos casos podemos afectar también al acero estructural también. Es aquí donde cobran importancia los ensayos no destructivos del concreto, que permiten estimar su calidad sin dañar la estructura del concreto; sin embargo y para el caso del esclerómetro, existe un vacío en el conocimiento que permite relacionar la resistencia del concreto obtenida mediante el ensayo de esclerometría y la resistencia real de una muestra de concreto para nuestra región, sobre todo estimar cual sería el margen de error y cuál es su porcentaje de confiabilidad.

Como antecedentes, existen algunas investigaciones a nivel mundial dentro de las cuales destacan las siguientes:

En la tesis de maestría presentada por el Ing. Jimenez, H (2010), para optar el grado de maestro en construcción en la Universidad mexicana Veracruzana, se concluye que existe una regresión lineal entre los resultados por esclerómetro y los resultados reales por compresión en prensa; y que el ensayo es factible y confiable. Al respecto y dentro de las probetas ensayadas con un esclerómetro mecánico encontró correlaciones $\left(\mathrm{r}^{2}\right)$ desde 0.8083 hasta 0.9266 .

Los ingenieros J.H. BUNGEY de la Universidad de Liverpool (Inglaterra) y R. MADANDOUST de la Universidad de Guilán (Irán) realizaron un estudio en el año (1997) "Evaluación de pruebas de resistencia no destructivas de concreto ligero", en la que concluyen que en sus ensayos de laboratorio se aprecia una variación de un $\pm 15 \%$ en el valor de la resistencia en mezclas iguales, así mismo recomiendan que para que los ensayos tengan mayor certeza los golpes con el esclerómetro deben haberse por ambas caras de las probetas cilíndricas o cúbicas. 
El Ingeniero F. HOSTALET, profesor asociado del Departamento de Ingeniería de la Construcción de la Escuela Técnica Superior de Caminos, Canales y Puertos de Barcelona; manifiesta que a nivel mundial tanto en Canadá, Australia, Japón y Estados Unidos, aún no contemplan la utilización de estas técnicas como instrumento de Control de Calidad durante la ejecución de una obra. Lo mismo sucede en Europa en general y Gran Bretaña.

Finalmente, en una investigación titulada "Correlación entre la resistencia del hormigón y las pruebas no destructivas combinadas para hormigón con cemento de alta resistencia inicial", realizada por Domingo R. (Far Eastern University, Filipinas) e Hirose S. (Instituto tecnológico de Tokio), encontraron que se establece una mejor correlación entre la estimación de la resistencia del concreto y un ensayo combinado de esclerometría (Q) y de velocidades de pulso de ultrasónicos (VPU).

En este sentido el objetivo de la presente investigación es encontrar el nivel de correlación entre la resistencia real del concreto $(\mathrm{kg} / \mathrm{cm} 2)$ y el ensayo no destructivo de esclerometría, para concretos de diferentes características de nuestra región, así mismo verificar como influye el nivel de fraguado y endurecimiento del concreto y determinar el espesor máximo de concreto hasta donde el ensayo de esclerometría es confiable.

La hipótesis que hemos planteado es que efectivamente si existe una correlación lineal fuerte entre el número de Rebote $(\mathrm{Q})$ del concreto obtenido mediante el ensayo no destructivo de esclerometría vs. la resistencia real a la compresión.

\section{Metodología}

Para realizar la investigación se ha evaluado una muestra de 331 probetas de concreto provenientes de diferentes obras de construcción en la región Lambayeque y también se han ensayado 27 probetas con diseño controlado para diferentes f'c: 175, 210 y $280 \mathrm{~kg} / \mathrm{cm} 2$; de esta manera hemos podido comparar en qué casos se tiene una mejor correlación: entre las probetas provenientes al azar de diferentes obras, o en las probetas de diseño controlado en laboratorio.

La muestra se ha calculado en base a una población finita estimada en función a la cantidad total de probetas que se están ensayando en todos los laboratorios de ensayo de materiales en la región durante los últimos 6 meses de la investigación. 
Los datos estadísticos son los siguientes:

$\mathrm{N}^{\circ}$ de laboratorios en la región: 05

Promedio de probetas rotas x mes: 45

$\mathrm{N}^{\circ}$ de meses de la investigación: 06

$\mathrm{N}($ Población $)=05 \times 45 \times 06=1350$ probetas

$\mathrm{p}($ probabilidad que la hipótesis sea cierta $)=50 \%$

$q=(1-p)=50 \%$

e $($ error máximo aceptable $)=5 \%$

$Z$ (coeficiente de confiabilidad 95\%) $=1.96$

$Z^{2}$.p.q. N

$\mathrm{n}=$

$$
\mathrm{e}^{2}(\mathrm{~N}-1)+\mathrm{Z}^{2} \cdot \mathrm{p} \cdot \mathrm{q}
$$

n= 299 probetas (muestreo mínimo)

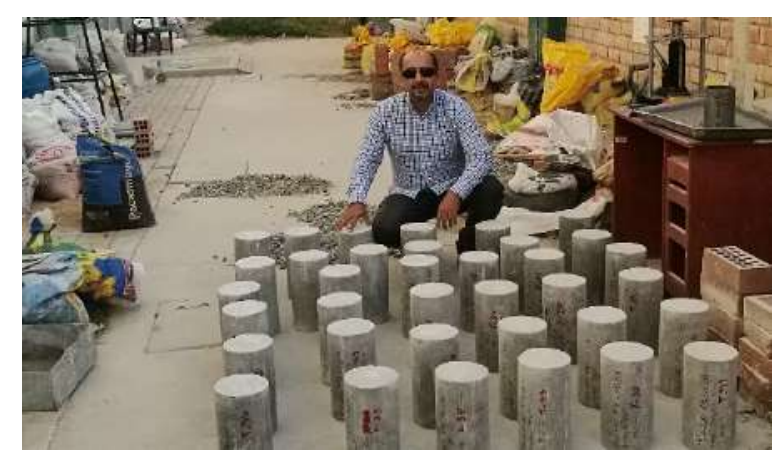

Foto 1: parte de las probetas a ser ensayadas 
El diseño de la investigación es de tipo descriptivo correlacional, siendo el modelo utilizado el siguiente:

$\mathrm{O}_{\mathrm{e}} \rightarrow \mathrm{M}_{1} \rightarrow \mathrm{M}_{2}$ : Para el grupo de testigos de las obras en ejecución

$\mathrm{O}_{\mathrm{c}} \rightarrow \mathrm{M}_{1} \rightarrow \mathrm{M}_{2}$ : Para el Grupo de testigos con diseños controlados ( $\mathrm{f}^{\prime} \mathrm{c}=175$, $210,280)$

Donde:

$\mathrm{O}_{\mathrm{e}}$ : Objeto de estudio: probeta

$\mathrm{M}_{1}$ : Medición del f’c con esclerómetro

$\mathrm{M}_{2}$ : Medición del f'c con prensa

El procedimiento seguido fue el siguiente:

$1^{\circ}$ Las probetas de diferentes obras se ingresaban al laboratorio previa codificación.

$2^{\circ} \mathrm{Se}$ tomaron medidas de las probetas: peso, diámetro y altura, verificando que las dimensiones cumplan con la norma técnica: $\mathrm{L} / \mathrm{D}=2$ y que la medida de dos diámetros no difieran en más de $2 \%$. Si la probeta no cumplía con estos requisitos se la excluía el ensayo.

$3^{\circ}$ Antes de realizar el ensayo de esclerometría se tenía que pulir la cara superior de las probetas y luego se aplicaba 10 impactos perpendiculares con el esclerómetro digital, según lo estipulado en la norma NTP 339.181 (2013) y ASTM C805 (2013). Si existían más de 2 lecturas que excedían en \pm 6 unidades el promedio $\left(\mathrm{Q}_{\mathrm{m}}\right)$ se descartaba el ensayo por su gran variabilidad. $\left(\mathrm{Q}_{\mathrm{m}}-6 \leq \mathrm{Q}_{\mathrm{i}} \leq \mathrm{Q}_{\mathrm{m}}+6\right)$

$4^{\circ}$ Se calculó el valor promedio Qm válido para esa probeta.

$5^{\circ}$ Enseguida se procedió a ensayar la probeta en la prensa hidráulica para obtener su verdadera resistencia a la compresión $\left(\mathrm{f}^{\prime} \mathrm{c}\right.$ real$)$.

$6^{\circ} \mathrm{El}$ procedimiento se repitió para todas las probetas de la muestra estadística. 


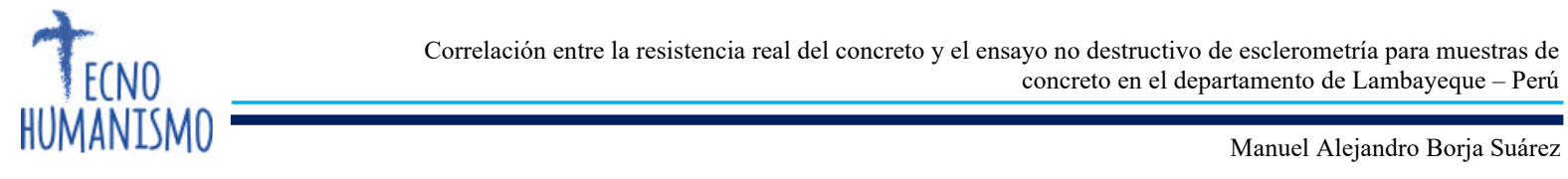

Los equipos que se utilizaron fueron los siguientes:

- 01 Esclerómetro Digital Silver Schmidth Tipo N, Marca PROCEQ - Nro. Serie: SH01-002-0509 con certificado de calibración vigente

- 01 Prensa Marca ELE ADL 1500 N$^{\circ}$ serie1796-8-1944 (Laboratorio USS).

- 01 Prensa marca Tamiequipos TCP 038, $\mathrm{N}^{\circ}$ serie 655 (Laboratorio A\&C Exploración Geotécnica y Mecánica de Suelos SRL).

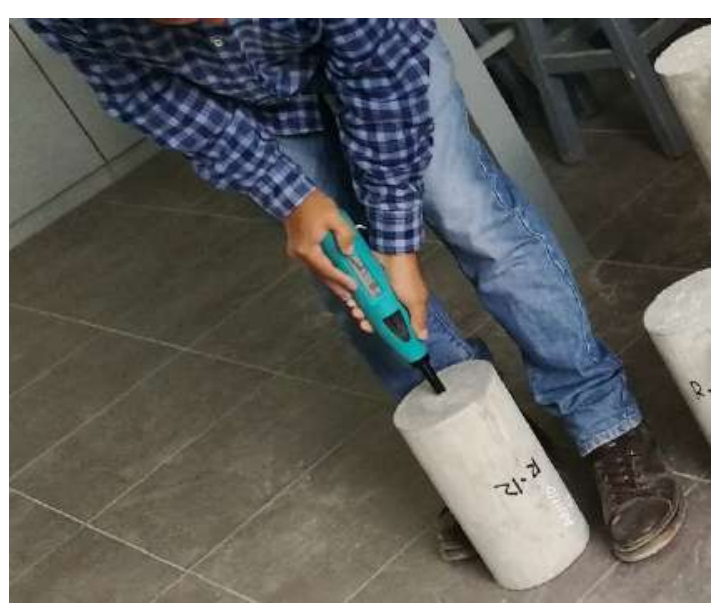

Foto 2: Ensayo de esclerometría

Una vez que se concluyeron los ensayos de laboratorio se obtuvieron las correlaciones estadísticas según los siguientes criterios:

- Comparación del f'c real del ensayo en la prensa hidráulica, con el f'c obtenido del esclerómetro según la ecuación de fábrica pre instalada en el equipo.

- Comparación del f'c real del ensayo en la prensa hidráulica, con el valor Q (adimensional) obtenido del esclerómetro.

Ambas comparaciones se hicieron para todas las probetas provenientes de diferentes obras y para las probetas de diseño controlado $\left(175,210\right.$ y $\left.280 \mathrm{kc} / \mathrm{cm}^{2}\right)$.

Los estadísticos principales que se obtuvieron fueron la desviación estándar, el coeficiente de correlación $\mathrm{r}^{2}$, la ecuación y el rango de estimación del f’c en función a un coeficiente de confiabilidad de $95 \%(Z=1.96)$. 


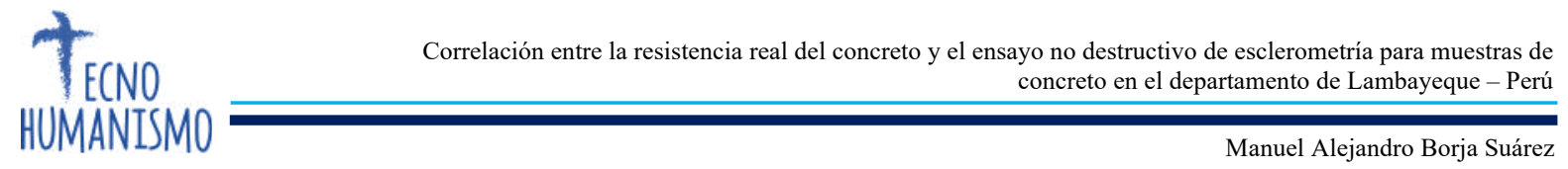

Así mismo se verificó cual sería el espesor máximo de concreto que el ensayo de esclerómetro podría detectar, para lo cual se utilizaron probetas de Cem:Arena (1:4) que incorporaban capas de mortero con esferas de poliestireno expandido (EPS) de espesor variable de 5, 10, 20 y $30 \mathrm{~cm}$. El supuesto era que el esclerómetro sería capaz detectar estas variaciones drásticas de dureza y resistencia, y arrojaría valores de Q diferentes para todas estas probetas, en caso contrario, si el esclerómetro era capaz de detectar esto, arrojaría resistencias similares para todas las probetas.

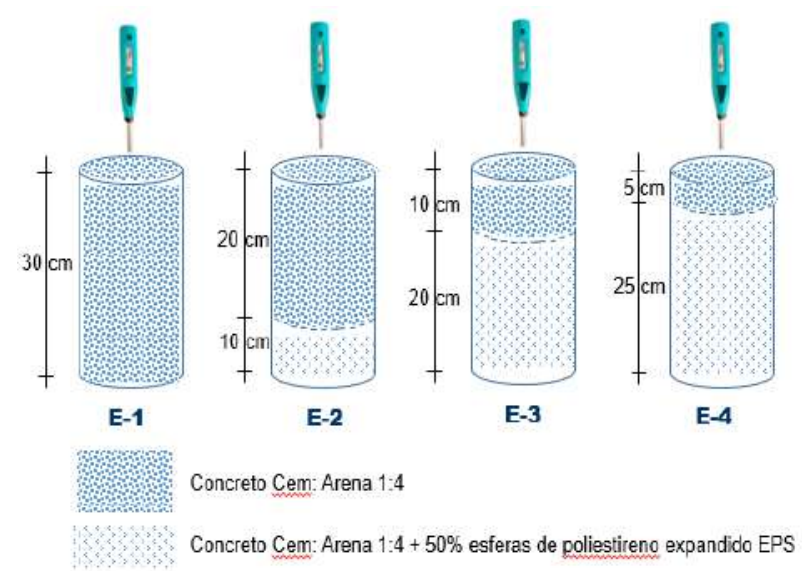

Foto 3: Probetas con concreto de diferentes densidades

\section{Resultados}

Por lo general los esclerómetros vienen con su propia ecuación de fábrica (impresa en un ábaco o grabada en el software del equipo), pero se debe tener en cuenta que estas ecuaciones solo son válidas para los países de origen; por esta razón los primero resultados de la investigación están relacionados con la confiabilidad de estas ecuaciones en nuestro país.

Se comparó el f'c calculado por el esclerómetro (con la ecuación de fábrica) vs. el f'c real de la probeta ensayada en la prensa, habiéndose obtenido una correlación baja de $\mathrm{r}^{2}=0.7249$. (ver gráfica $\mathrm{N}^{\circ} 1$ ) 


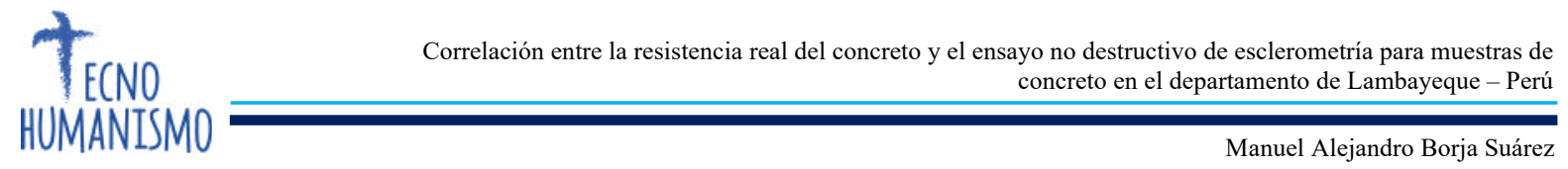

Gráfica $N^{\circ}$ 1. Correlación entre f'c del equipo vs. f'c Real (Utilizando Ec. De fábrica)

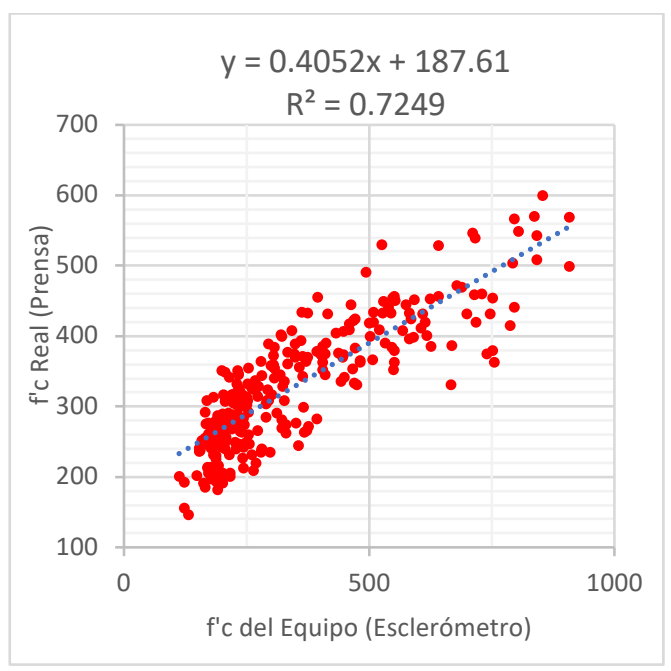

Así mismo, al utilizar esta ecuación de fábrica del esclerómetro para estimar el f'c del concreto se obtuvieron errores de hasta el 100\% al compararlos con el f'c real de la prensa; lo que sugiere que no se debe utilizar directamente estas ecuaciones de fábrica para estimar la resistencia del concreto en nuestra región Lambayeque, ya que estas ecuaciones vienen calibradas para los concretos de los países fabricantes de los equipos. (ver gráfica $\mathrm{N}^{\circ} 2$ )

La segunda correlación se obtuvo al comparar el valor Q (adimensional) del esclerómetro vs. el f'c real de las probetas ensayadas en la prensa, habiéndose obtenido la siguiente ecuación de correlación. (ver gráfica $\mathrm{N}^{\circ} 3$ )

$y=10.17 x-147.22 \ldots\left(r^{2}=0.7437\right)$

Gráfica $\mathrm{N}^{\circ}$ 2. Errores de estimación del f'c, utilizando Ecuación de fábrica)

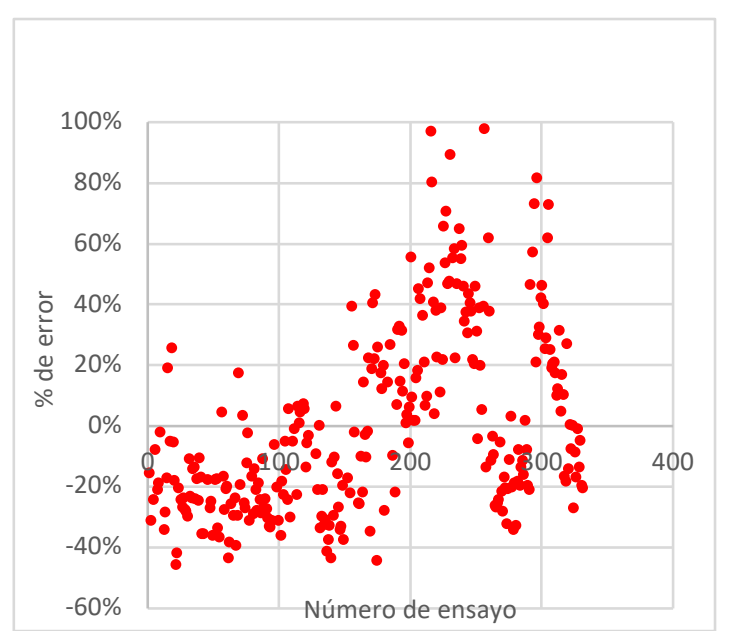


Gráfica $\mathrm{N}^{\circ}$ 3. Correlación entre el número de rebote $(\mathrm{Q})$ vs. f'c real de las probetas

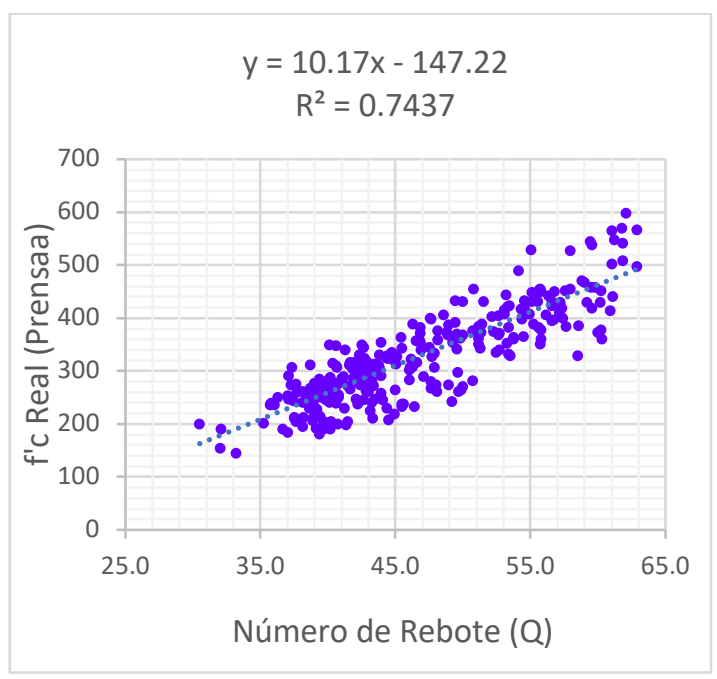

Como se observa la correlación no es muy alta, debido a la gran variabilidad de concretos evaluados, que provenían de diferentes obras de la región, lo que implicaban diferentes canteras de agregados, diferentes granulometrías y dosificaciones.

Para el caso de concretos de diseño controlado, la correlación mejoró considerablemente sobre todo cuando las probetas cumplían su ciclo completo de curado, así se obtuvieron los siguientes resultados:

-Probetas a los 7 días:

$\mathrm{r} 2=0.726$

-Probetas a los 14 días:

$\mathrm{r} 2=0.8446$

-Probetas a los 28 días:

$$
\mathrm{r} 2=0.9467
$$

Esta variación significativa de correlación se debe principalmente la diferencia de dureza del concreto y contenido de humedad interno entre una probeta de 7 días de curado y otra de 28 días.

En base a esto, se correlacionó solo los resultados de probetas ya endurecidas a los 28 días, habiéndose obtenido la siguiente ecuación (ver gráfica $\mathrm{N}^{\circ} 4$ )

$$
\mathrm{f}^{\prime} \mathrm{c}=11.05 \mathrm{Q}-257.26\left(\mathrm{r}^{2}=0.9467\right)
$$

Este resultado significa que cualquier medición hecha con el esclerómetro tiene un 94.67\% de probabilidad de ser estimada con esta ecuación. 
Así mismo, con esta ecuación local se volvieron a calcular los f'c teóricos y así determinar el error de estimación (Diferencia entre f'c de la prensa - f'c ecuación local). De acuerdo a esto, se obtuvo una desviación estándar $(\sigma)$ de los errores de estimación, de $8.8 \mathrm{~kg} / \mathrm{cm} 2(3.7 \%)$

Esto significa que para un nivel de confianza del 95\% (1.96 $\sigma)$ se puede inferir que las medidas hechas con el esclerómetro estarían en un rango de f'c $\pm 17.3 \mathrm{~kg} / \mathrm{cm} 2 .( \pm$ $7.2 \%)$.

Gráfica $\mathrm{N}^{\circ}$ 4. Correlación entre $\mathrm{N}^{\circ}$ de rebote (Q) vs. f'c (real) (probetas a 28 días)

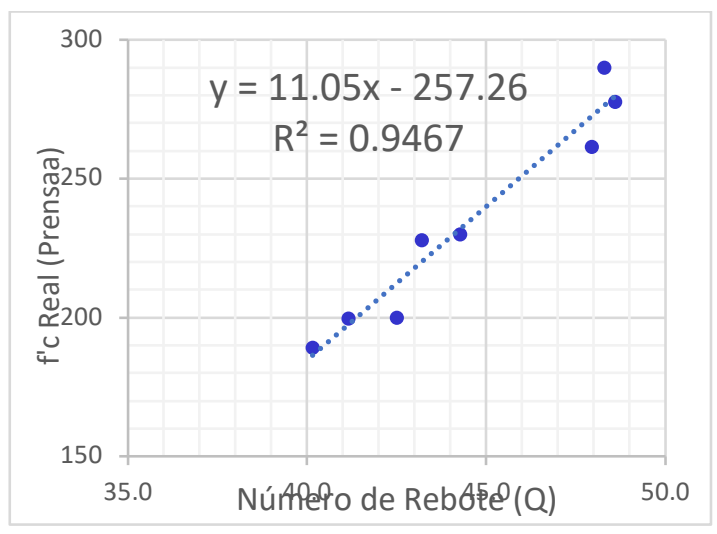

Para el análisis del espesor máximo de concreto que es detectado por el esclerómetro, se obtuvieron los siguientes resultados:

Gráfica $\mathrm{N}^{\circ}$ 5. Ensayo de esclerometría en probetas con capas de densidades diferentes.

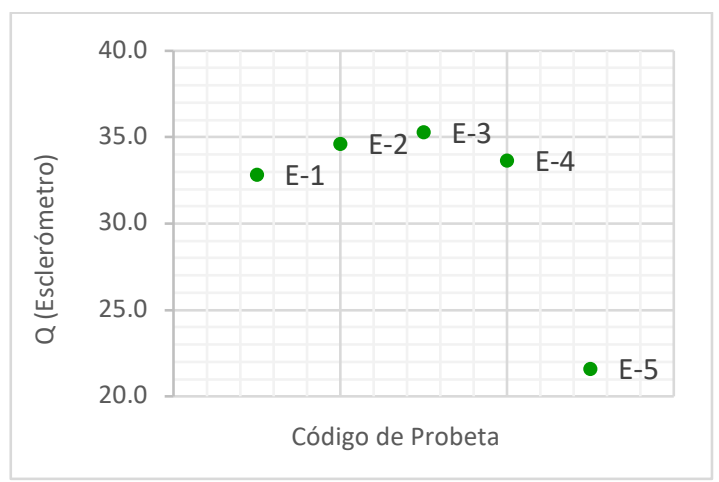

Donde:

E1: Probeta $30 \mathrm{~cm}$ concreto Cem:Arena 
E2: Probeta $20 \mathrm{~cm}$ concreto $+10 \mathrm{~cm}$ EPS

E3: Probeta $10 \mathrm{~cm}$ concreto $+20 \mathrm{~cm}$ EPS

E4: Probeta $5 \mathrm{~cm}$ concreto $+25 \mathrm{~cm}$ EPS

E5: Probeta $100 \%$ mortero + EPS

Según se puede observar, el esclerómetro mostró casi los mismos valores Q para probetas con capas de concreto de 5, 10, 20 y $30 \mathrm{~cm}$, lo que significaría que el ensayo de esclerometría puede determinar la dureza del concreto solamente de los primeros $5 \mathrm{~cm}$ de profundidad.

\section{Discusión}

A diferencia de otras investigaciones que han encontrado correlaciones entre el esclerómetro y el ensayo a compresión del concreto, todas las han hecho solamente con probetas de diseño controlado; en nuestro caso se evaluaron más de 300 probetas al azar provenientes de diferentes obras, lo que significaba que nuestros resultados podrían ser utilizados de manera general para cualquier tipo de obra. Sin embargo al tratar de correlacionar todos estos valores con una sola ecuación, el coeficiente de correlación resultó ser bajo $\left(\mathrm{r}^{2}=0.7437\right)$.

Sin embargo, para el concreto de diseño controlado, se encontró resultados similares a los obtenidos por otros autores como SUAREZ, E. (2013) y JIMENEZ, H.; cuyas investigaciones también encontraron correlaciones superiores a 0.9 (Ver Gráfica $\mathrm{N}^{\circ} 4$ ); siendo el error de los resultados de solo 7.2\%, para un nivel de confiabilidad del 95\%, que es muy inferior al obtenido por otros investigadores como DELIBES, A. (1993) y BUNGEY, J. (1997).

Otro aspecto por considerar es que se demostró, al igual que ROJAS, R. (2010) y BUILES, R.; que la humedad del concreto afecta al número de rebote del esclerómetro, y que los resultados en probetas que todavía no han cumplido su tiempo de fraguado ( $7 \mathrm{y}$ 14 días) tienen mucha dispersión y correlaciones muy bajas; lo que significa que un ensayo de esclerometría necesariamente deberá hacerse como mínimo cuando el concreto haya endurecido a los 28 días. 
Uno de los aspectos que queda pendiente para una investigación complementaria es determinar el espesor exacto de concreto hasta donde el esclerómetro es capaz de estimar su dureza y resistencia, al respecto en la presente investigación se encontró que sería solo de $5 \mathrm{~cm}$ de concreto como máximo, sin embargo, este valor podría ser ajustado para rangos aún menores y con un mayor número de testigos.

\section{Conclusiones}

- Las ecuaciones de fábrica con las que vienen configurados los esclerómetros no son válidas ni confiables para nuestro país, teniendo que encontrarse una ecuación de correlación en cada región donde se tengan que utilizar.

- La correlación encontrada entre el valor Q (medido por el esclerómetro y el f'c (obtenido en la prensa hidráulica) para diferentes tipos de concreto (331 testigos de diferentes obras, canteras y dosificaciones), fue baja $\left(\mathrm{r}^{2}=0.7437\right)$ esto debido a la gran variabilidad de sus agregados y procesos propios de obra. (Ver figura $\mathrm{N}^{\circ}$ 16)

- Sin embargo, en las probetas de diseño controlado (una misma cantera y f'c: 175 , 210 y $280 \mathrm{~kg} / \mathrm{cm}^{2}$ ) que completaron su periodo de fraguado y endurecimiento a 28 días, la correlación fue muy buena $\left(\mathrm{r}^{2}=0.9467\right)$, siendo la ecuación de predicción, la siguiente:

$f^{\prime} c=11.05 Q-257.26$

Para este caso, se obtuvo una desviación estándar de $8.8 \mathrm{~kg} / \mathrm{cm}^{2}$ y teniendo en cuenta un nivel de confiablidad del 95\% el f’c del concreto estimado con el esclerómetro, podría encontrarse dentro de este margen de error:

$$
\mathrm{f}^{\prime} \mathrm{c} \pm 17 \mathrm{~kg} / \mathrm{cm}^{2} \text { o f'c } \pm 7.2 \%
$$

- Con respecto a la humedad interna de un concreto y el proceso de fraguado, se concluye que estas afectan significativamente al ensayo del esclerómetro, habiéndose obtenido una mejor correlación en los testigos que habían cumplido su proceso de fraguado y endurecimiento a los 28 días; las correlaciones que se obtuvieron así lo demuestran:

- Probetas ensayadas a los 7 días:

$\mathrm{r} 2=0.726$ 
- Probetas ensayadas a los 14 días:

$$
\mathrm{r} 2=0.8446
$$

- Probetas ensayadas a los 28 días:

$$
\mathrm{r} 2=0.9467
$$

Por esta razón se recomienda que el ensayo de esclerometría se aplique en elementos de concreto endurecido como mínimo a los 28 días.

- El esclerómetro solo detecta la dureza y resistencia hasta los primeros $5 \mathrm{~cm}$ de profundidad de una estructura de concreto, limitante que debe tenerse en cuenta al momento de analizar las estructuras.

- Como conclusión final, podemos manifestar que el ensayo de esclerometría si se puede utilizar para estimar la resistencia a la compresión del concreto pero bajo ciertas condiciones controladas siempre y cuando se indique también el nivel de confiabilidad y el margen de error estadístico.

\section{Referencias bibliográficas}

ASTM C805 (2013). Standard Test Method for Rebound Number of Hardened Concrete

AVID, F.; SAAD, J; SOTA J. (2010). Estudio del hormigón de una estructura expuesta a la intemperie por quince años, construida en dos etapas, VI Congreso internacional sobre patología y recuperación de estructuras. Argentina.

BUILES, R. y PARDO, M. (2016). Tesis “Correlación entre las resistencias obtenidas mediante ensayos de compresión y esclerometría en cilindros de concreto normal y modificados con fibra sintética y fibra de acero". Universidad Católica de Colombia.

BUNGEY, J. (1997) Pruebas no destructivas en Ingeniería Civil, Instituto Británico de Inglaterra.

CESPEDES, M (2003). Tesis para optar el grado de Ingeniero Civil: "Resistencia al a compresión del concreto a partir de la velocidad de pulsos de ultrasonido". Universidad de Piura.

DAMAZO, J (2006). Artículo: “Las estructuras no son eternas”. Revista: Construcción y tecnología. YMCYC. 
DELGADO, F. \& GUERRERO, (2015) Tesis: “Correlación entre el número de rebote (NTC 3692) y la resistencia a la compresión (NTC 673) en cilindros de concreto utilizando materiales de la mina El Espino y las canteras Pabón y Pilcuan2. Universidad de Nariño, Colombia.

DELIBES, A (1982). Determinación de la resistencia del hormigón mediante ensayos no destructivos realizados con esclerómetro y ultrasonidos. Revista virtual Informes de la Construcción Vol 33, $\mathrm{N}^{\circ}$ 338. Acceso: 15/10/2016. Disponible en: http://informesdelaconstruccion.revistas.csic.es/index.php/informesdelaconstrucci on/article/view/2141

DOMINGO R, \& HIROSE S (2004). Investigación "Correlation between concrete strength and combined nondestructive tests for concrete using high-early strength cement", Far Eastern University, Filipinas

EN 12504-2 (Norma Europea) Procedimiento para determinar el número de rebote

HERNANDEZ, Roberto (2003). Metodología de la Investigación, 3ra Edición, Edit. McGraw Hill, México

HOSTALET, F. (1994). Artículo "Situación actual de las técnicas de ensayo no destructivo del hormigón”, Escuela técnica superior de ingeniero de caminos, canales y puertos. Barcelona.

IMCYC (2009). Determinación del índice de rebote utilizando el dispositivo conocido como esclerómetro. Instituto Mexicano del Cemento y del Concreto. Acceso: 30Set-2016. Disponible en: http://www.imcyc.com/ct2009/mar09/problemas.pdf

INDECOPI (2013). CONCRETO. Método de ensayo para determinar el número de rebote del concreto endurecido (esclerometría) NTP. 339.181.2013. 2da. Edición. Lima.

INDECOPI (2008). CONCRETO. Método de ensayo normalizado para la determinación de la resistencia a la compresión del concreto en muestras cilíndricas. NTP. 339.034.2008. 3ra. Edición. Lima.

JIMENEZ, H. (2010), Tesis Maestría: “Correlación de la resistencia a la compresión de cilindros de concreto hidráulico con el ensayo esclerométrico". Universidad Veracruzana. México 
QUEZADA, N. (2010) Metodología de la Investigación: estadística aplicada a la investigación. Editorial Macro, 1ra. Edic. Lima Perú.

ROJAS, R. (2010) Tesis “Determinación de la resistencia a la compresión de cilindros a edades tempranas mediante el empleo del esclerómetro”. Universidad Veracruzana. México.

SAN JUAN, C. (2013) Resistencia del hormigón mediante esclerómetro o índice de rebote. Publicado en Revista virtual Patología + Rehabilitación + Construcción. Acceso: 19-Set-2016. Disponible en: https://www.patologiasconstruccion.net.

SUAREZ, E. (2013) Tesis "Estudio comparativo de la resistencia a compresión de elementos de concreto obtenida por rotura de cilindros y con martillo o esclerómetro digital". Faculta de Ingeniería, Instituto Tecnológico de Santo Domingo. República Dominicana.

Resistencia del hormigón mediante esclerómetro o índice de rebote (1) Acceso 04/05/2018. https://www.patologiasconstruccion.net/2013/11/resistencia-delhormigon-mediante-esclerometro-o-indice-de-rebote-1/

Resistencia del hormigón mediante esclerómetro (2) Ensayo y valor Fc. Acceso 05/Jun/2018. 